\title{
PDGFRA Gene Rearrangement
}

National Cancer Institute

\section{Source}

National Cancer Institute. PDGFRA Gene Rearrangement. NCI Thesaurus. Code C84271.

Rearrangement of the PDGFRA gene, resulting in the formation of a fusion transcript and aberrant tyrosine kinase activity. It has been described in hematologic malignancies usually presenting as chronic eosinophilic leukemia. 